\title{
Rendements comparés
} en muscles consommables, os, graisse, aponévroses après transformation classique (coupe de Paris)
d'une demi-carcasse de N'Dama et de Zébu

par R. LETROTEUR

Cette étude a pour but, essentiellement pratique, de connaître le rendement en viande des carcasses de bovins, destinées aux fournitures de l'armée ou aux collectivités administratives.

En Afrique Occidentale Française, les animaux étant très rarement pesés sur pied, nous avons délibérément laissé de côté le poids vif, ce facteur n'intervenant pas dans la pratique.
La « carcasse type » de zébu, choisie pour effectuer la découpe, pesait $158 \mathrm{~kg}$ sans hampe, ni onglet, ni rognons et provenait d'un bœuf de race Peule, originaire du Soudan et âgé de 8 ans environ.

La carcasse de N'Dama pesait $134 \mathrm{~kg}$ sans hampe, ni onglet, ni rognons et provenait d'un bœuf originaire de Bouaké, descendant de N'Dama dé race pure, importés d'Odionné, et âgé de 7 ans.

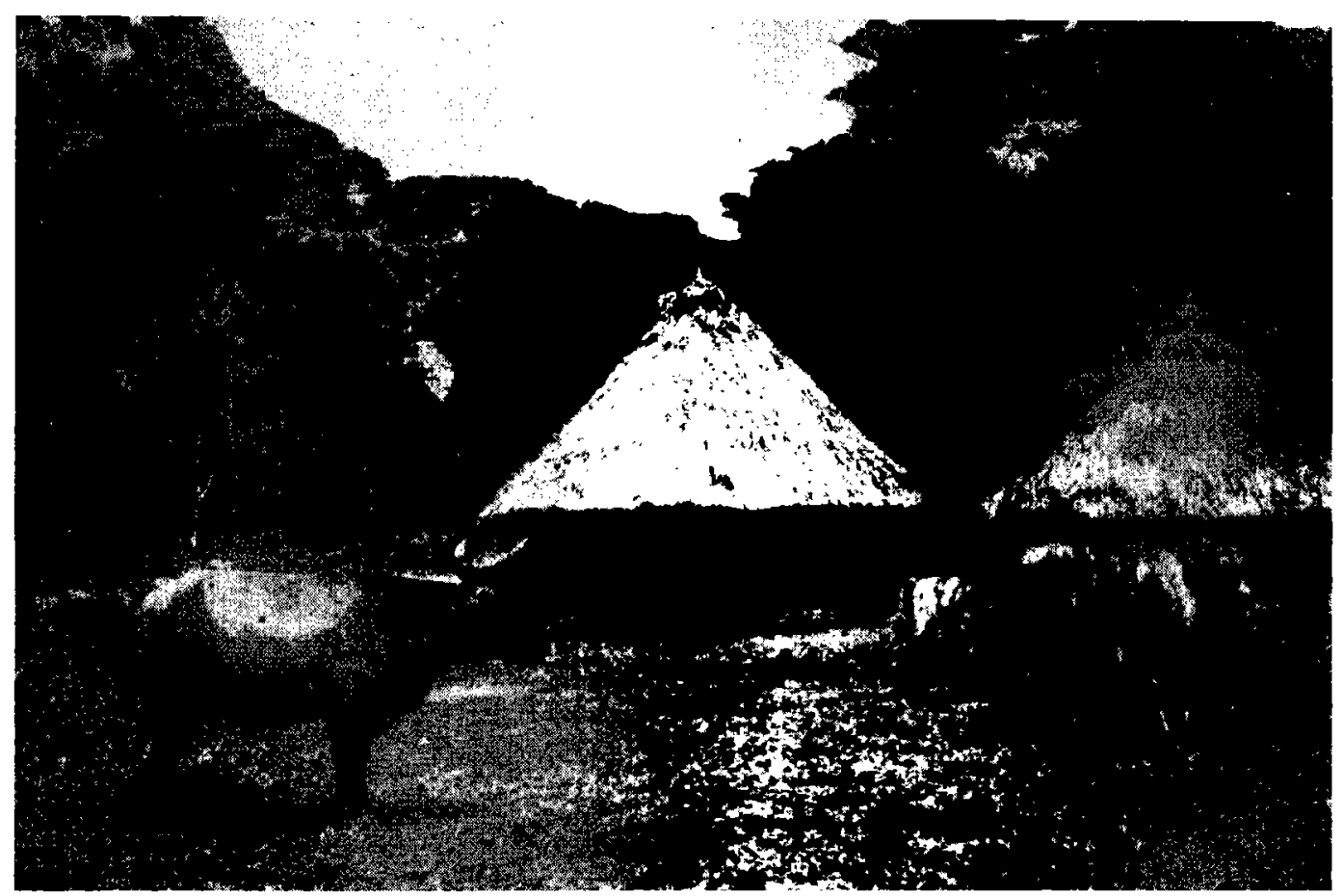


TABLEAU I.

\section{CARCASSE DE N'DAMA}

Poids de la demi-carcasse côté queue, sans hampe ni onglet : $67,160 \mathrm{~kg}$

\begin{tabular}{|c|c|c|c|c|c|c|}
\hline \multirow{2}{*}{ DEETAIL DES MORCEAUX } & \multirow{2}{*}{$\begin{array}{c}\text { Poids } \\
\text { après } \\
\text { découpage }\end{array}$} & \multicolumn{2}{|c|}{$\begin{array}{c}\text { Poids des muscles } \\
\text { consommables }\end{array}$} & \multirow{2}{*}{$\begin{array}{l}\text { Poids } \\
\text { des os }\end{array}$} & \multirow{2}{*}{$\begin{array}{l}\text { Poids } \\
\text { graisse }\end{array}$} & \multirow{2}{*}{$\begin{array}{l}\text { Poids de } \\
\text { aponévroses }\end{array}$} \\
\hline & & $\begin{array}{l}\text { à rôtir } \\
\text { ou à griller }\end{array}$ & $\begin{array}{l}\text { à bouillir } \\
\text { ou à braiser }\end{array}$ & & & \\
\hline $\begin{array}{l}\text { 1. Cuisse - Poids total } 17,920 \\
\text { Jambe } \ldots \ldots \ldots \ldots \ldots \ldots\end{array}$ & 3,270 & - & 1,620 & 1,440 & - & 0,210 \\
\hline Tende de tranche avec coxal & 5,940 & 4,760 & - & 0,820 & 0,100 & 0,260 \\
\hline Gîte à la noix $\ldots \ldots \ldots \ldots$. & 3,110 & 2,810 & - & - & 0,050 & 0,250 \\
\hline Tranche grasse avec os.... & 4,490 & 2,710 & - & 1,590 & - & 0,190 \\
\hline Nerveux de gîte........... & 1,110 & - & 0,920 & - & - & 0,190 \\
\hline $\begin{array}{l}\text { 2. Aloyau - Poids total } 10,170 \text {. } \\
\text { Filet }\end{array}$ & 1.560 & 1,380 & - & - & 0,050 & 0,130 \\
\hline Faux-filet $\ldots \ldots \ldots \ldots \ldots$ & 4,060 & 2,600 & - & 1,020 & - & 0,440 \\
\hline $\begin{array}{l}\text { Rumsteack avec aiguillette, } \\
\text { baronne et araignée ...... }\end{array}$ & 4,550 & 2,900 & 0,720 & 0,320 & 0,150 & 0,460 \\
\hline 3. Train de côtes - & & 4030 & & 770 & & 0320 \\
\hline Surlonge . . . . . ........ & 2,980 & - & 2,160 & 0,610 & - & 0,210 \\
\hline 4. Plat de côtes ............. & 3,500 & -- & 2,230 & 0,820 & - & 0,450 \\
\hline $\begin{array}{l}\text { 5. Bavette d'aloyau-Pot-au-feu } \\
\text { et beefsteack ............. }\end{array}$ & 4,240 & 0,730 & 2,470 & 0,170 & 0,680 & 0,190 \\
\hline 6. Pis & 6,020 & - & 4,230 & 1,250 & 0,350 & 0,190 \\
\hline $\begin{array}{l}\text { 7. Paleron - Poids total } 10,340 \text {. } \\
\text { Jambe }\end{array}$ & 1,470 & - & 0,680 & 0,680 & 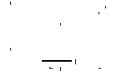 & 0,110 \\
\hline Charolaise $\ldots \ldots \ldots \ldots \ldots$ & 1,410 & - & 0,800 & 0,530 & $i$ & 0,80 \\
\hline Jumeaux à beefsteack & 0,720 & 0,640 & - & - & 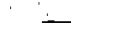 & 0,80 \\
\hline $\begin{array}{l}\text { Macreuse à beefsteack } \ldots \\
\text { Jumeaux nerveux, griffe, ta- } \\
\text { lon, macreuse, derriere de } \\
\text { paleron } \ldots \ldots \ldots \ldots \ldots \ldots\end{array}$ & 1,410 & 1,160 & 3,690 & 1,330 & 0,120 & 0,310 \\
\hline 8. Collier $\ldots \ldots \ldots \ldots \ldots \ldots$. & 4,240 & - & 3,140 & 0,890 & - & 0,210 \\
\hline 9. Queve ..... & 1,040 & - & 0,800 & $\begin{array}{l}\text { ne se dé- } \\
\text { sosse pas }\end{array}$ & - & 0,240 \\
\hline $\begin{array}{l}\text { 10. Graisse } \\
\text { « rognons et bourses ».. }\end{array}$ & 1,230 & - & - & - & 1,230 & - \\
\hline TOTAL .............. & 67,160 & 23,720 & 23,460 & 12,610 & 2,730 & 4,640 \\
\hline POURCENTAGES. & $100 \%$ & $\begin{array}{r}35,3 \% \\
70\end{array}$ & $\begin{array}{l}34,9 \% \\
\%\end{array}$ & $18,8 \%$ & $4,1 \%$ & $6,9 \%$ \\
\hline
\end{tabular}




\section{TABLEAU IT}

\section{CARCASSE DE ZÉBU}

Poids de la demi-carcasse côté queue, sans hampe ni onglet : 79,410 kg

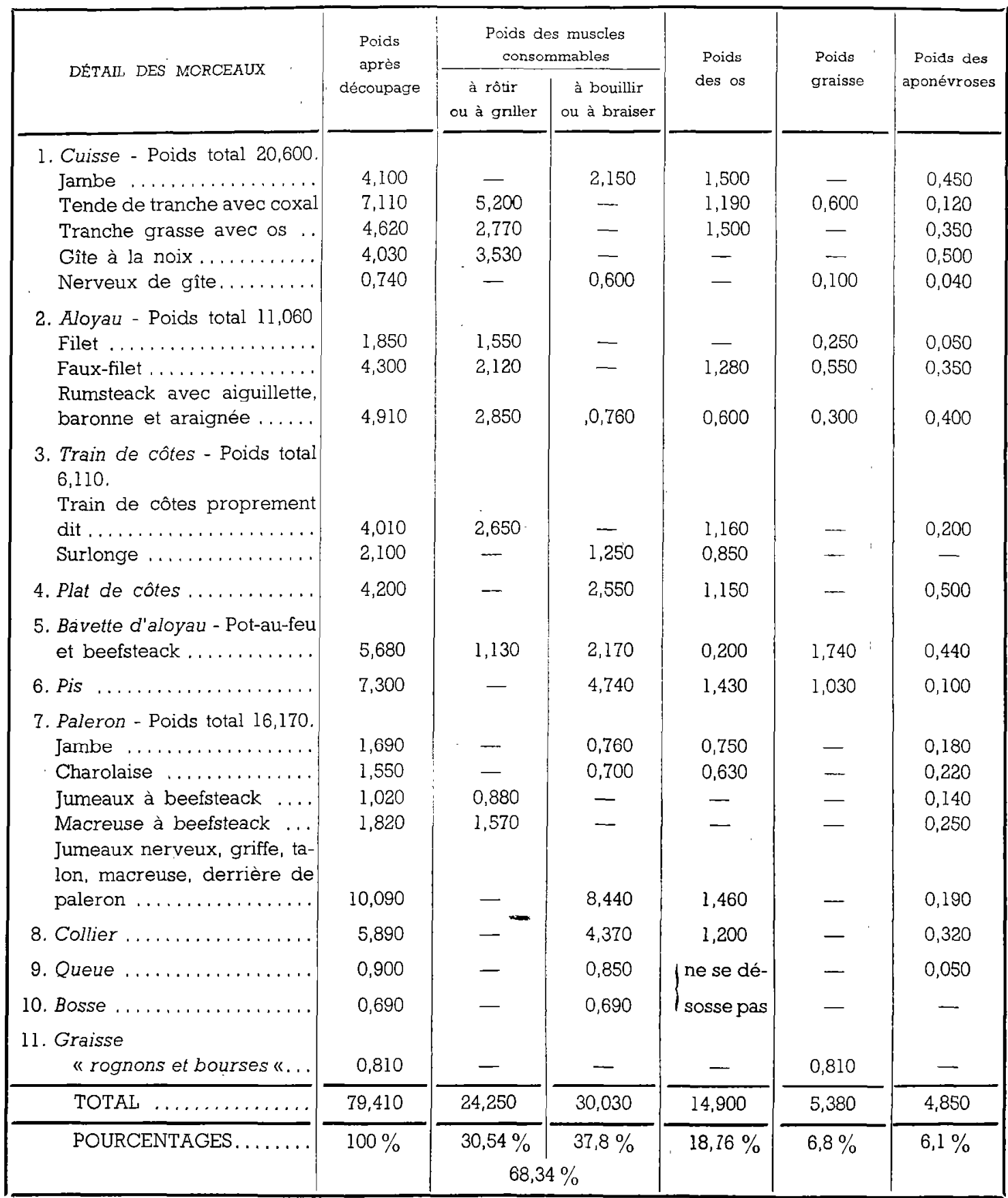


Ces bovins étaient en bon état d'embonpoint et de bonne conformation moyenne, telle que celle-ci est définie par tous les auteurs, en particulier par G. Doutressoulle dans son étude sur la morphologie générale de la race zébu et N'Dama (Elevage en A.O.F.).

Les résultats des pesées sont consignés dans les deux tableaux ci-dessous.

Ces pesées ayant été effectuées quelques heures après l'abatage de l'animal, nous n'avons pas tenu compte des pertes par dessiccation, qui n'entrent
Viandes à rôtir ou à griller.............. 35

Viandes à bouillir ou à braiser avec os...... 42

Gros os ....................... 10

Total ................. 87

soit un rendement approximatif de $70 \%$ en considérant que le pourcentage d'os varie entre 16 et $18 \%$.

\section{CONCLUSION}

Ces deux carcasses de bonine conformation

\begin{tabular}{|c|c|c|c|c|c|c|}
\hline & $\begin{array}{l}\text { Poids } \\
\text { après } \\
\text { découpe }\end{array}$ & $\begin{array}{c}\text { Viande } \\
\text { à rótir } \\
\text { ou à griller }\end{array}$ & $\begin{array}{c}\text { Viande } \\
\text { à bouillir } \\
\text { ou à braiser }\end{array}$ & Os & Graisse & Déchets \\
\hline \multirow{2}{*}{ ZÉBU } & \multirow{2}{*}{100} & 30,54 & 37,8 . & \multirow{2}{*}{18,76} & \multirow{2}{*}{6,8} & \multirow{2}{*}{6,1} \\
\hline & & \multicolumn{2}{|c|}{68,34} & & & \\
\hline \multirow{2}{*}{ N'DANA } & \multirow{2}{*}{100} & 35,3 & 34,9 & \multirow{2}{*}{18,8} & \multirow{2}{*}{4,1} & \multirow{2}{*}{6,9} \\
\hline & & & & & & \\
\hline
\end{tabular}

pas dans cette étude et qui sont d'ailleurs variables suivant les climats, et en particulier, suivant l'état hygrométrique de la région considérée.

La lecture des tableaux I et II montre des différences de rendement importantes dans la répartition des viandes de première et deuxième catégories :

A titre de comparaison, le détail de la découpe de Paris, retenue en France pour le bœuf, pour l'établissement du barème, publié en annexe de l'arrêté 22.595 du 20 octobre 1953 est le suivant pour $100 \mathrm{~kg}$ de carcasse : moyenne ont des rendements en viande comparables à des carcasses de bovins de la métropole.

Chez le N'Darna, le pourcentage en viande de première catégorie est absolument identique. Chez le Zébu, où l'on constate un développement moindre des régions " aloyau et cuisse », ce rendement est beaucoup plus faible. Enfin, pour terminer, signalons que la graisse apparemment en moins grande quantité chez le N'Dama est en réalité beaucoup mieux répartie (viande marbrée) que chez le Zébu où cette graisse. se concentre seulement à certains endroits de la carcasse.

\section{Comparison of the carcass yields of a N'Dama Bovine and of a zebu.}

The writer has compared the weights of edible meat, of bone, fat, and aponeur osis, from the carcasses of a peuhl zebu, eight years old, and a N'Dama ox, seven years old.

The results of these observations are tabulated. They show that these two animals have a meat yield which can be compared with those of French bovines, but in the zebu the proportion of meat of the first category is lower and the fat is not evenly distributed.

\section{RESUMEN}

Comparación del rendimiento en carne de un bovino N'Dama y de un cebú.

El autor ha comparado las cantidades de carne para consumo; huesos, grasa, aponeurosis, suministradas por un cebú peuhl, de 8 años y las de un buey N'Dama, de 7 años.

Los resultados de estas observaciones pueden verse en diferentes tablas. Demuestran que estos dos animales tienen rendimientos en carne comparables a los de los bovinos franceses.

Pero en el cebú, la proporción de carne de primera calidad es menor y la grasa no está uniforemmente repartida. 